\title{
Performance Limits on Ranging with Cognitive Radio
}

\author{
Davide Dardari*, Yasir Karisan ${ }^{\sharp}$, Sinan Gezici ${ }^{\sharp}$, Antonio A. D’Amico ${ }^{\dagger}$, and Umberto Mengali ${ }^{\dagger}$ \\ * WiLAB-DEIS, University of Bologna at Cesena, via Venezia 52, 47023 Cesena (FC), Italy \\ $\sharp$ Department of Electrical and Electronics Engineering, Bilkent University, Bilkent, Ankara 06800, Turkey \\ $\dagger$ Department of Information Engineering of the University of Pisa, Via Caruso 16, Pisa, Italy \\ Emails: ddardari@ieee.org, \{ykarisan,gezici\}@ee.bilkent.edu.tr, \{antonio.damico,umberto.mengali\}@iet.unipi.it
}

\begin{abstract}
Cognitive radio is a promising paradigm for efficient utilization of the radio spectrum due to its capability to sense environmental conditions and adapt its communication and localization features. In this paper, the theoretical limits on time-of-arrival estimation for cognitive radio localization systems are derived in the presence of interference. In addition, an optimal spectrum allocation strategy which provides the best ranging accuracy limits is proposed. The strategy accounts for the constraints from the sensed interference level as well as from the regulatory emission mask. Numerical results are presented to illustrate the improvements that can be achieved by the proposed approach.
\end{abstract}

Index Terms - Ranging, time-of-arrival (TOA) estimation, cognitive radio, interference, ultra-wideband (UWB), orthogonal frequency division multiplexing (OFDM).

\section{INTRODUCTION}

Cognitive radio $(\mathrm{CR})$ is an emerging paradigm that provides more efficient and flexible usage of the radio spectrum in the presence of coexisting heterogeneous technologies such as positioning and communication systems [1], [2], [3]. The basic idea is that a CR terminal can sense the environment and can adapt its features (such as power, frequency, modulation, etc.) so as to allow the dynamic reuse of the available spectrum [2]. The CR concept may also be applied in the context of high-definition location systems where the accuracy in ranging (hence, in localization) can be varied according to the available bandwidth (cognitive positioning systems) [4], [5].

Ultrawide bandwidth (UWB) technology is a viable candidate for enabling accurate localization capabilities due to its ability to resolve multipath propagation and penetrate obstacles [6], [7]. Its main feature is a fine delay resolution property that is exploited for estimating the time-of-arrival (TOA) of the first path signal [8], [9]. Especially in its multicarrier version [10], UWB is also well-suited for CR applications as an underlay technology thanks to its intrinsic capability to perform wideband sensing and adapt its transmitted spectrum shape.

In this paper, we first analyze the Cramér-Rao bound (CRB) for TOA estimation in the presence of interference due, for example, to one or more communication systems sharing the same spectrum. Second, we determine the optimal power allocation scheme (i.e., transmitted signal spectrum shape) that minimizes the $\mathrm{CRB}$ under constraints coming both from the transmitted signal spectrum mask and the sensed interference spectrum. Finally, numerical examples are provided and concluding remarks are made.

\footnotetext{
${ }^{0}$ This research was supported in part by the European Commission in the framework of the FP7 Network of Excellence in Wireless COMmunications NEWCOM++ (contract no. 216715) and in part by the FP7 European Project EUWB (Grant no. 215669).
}

\section{SignAl MODEL}

Due to their flexibility in utilizing the radio spectrum, multicarrier signals are commonly employed in cognitive radio systems [11]. In this paper, we adopt a signaling scheme of this type and we model the transmitted baseband signal over the symbol period $0 \leq t \leq T_{\mathrm{s}}$ as ${ }^{1}$

$$
s(t)=\sum_{k=1}^{K} \sqrt{w_{k}} p(t) e^{\jmath 2 \pi f_{k} t},
$$

where $f_{k}=(k-K / 2) \Delta$ is the $k$ th subcarrier frequency shift with respect to the center frequency, $\Delta$ is the subcarrier spacing, and $p(t)$ is a pulse with duration $T_{\mathrm{s}}$ and energy $E_{\mathrm{p}}$. The weights $w_{k} \geq 0$ permit spectrum shaping under the constraint $\sum_{k=1}^{K} w_{k}=1$ so that $E_{\mathrm{p}}$ and $P_{\mathrm{t}}=E_{\mathrm{p}} / T_{\mathrm{s}}$ are, respectively, the energy and the power of the baseband signal. ${ }^{2}$ In practice, the weights $w_{k}$ are also limited by peak power constraints, as will be shown in Section IV when determining the optimal transmitted signal spectrum.

Assuming that $\Delta$ is small enough compared to the channel coherence bandwidth, the baseband received signal corresponding to (1) is given by

$$
r(t) \cong s_{\mathrm{r}}(t-\tau)+n(t)
$$

with

$$
s_{\mathrm{r}}(t)=\sum_{k=1}^{K} \alpha_{k} \sqrt{w_{k}} p(t) e^{\jmath 2 \pi f_{k} t}
$$

where $\tau$ is the propagation delay, $\alpha_{k}=a_{k} e^{\jmath \phi_{k}}$ represents the complex channel coefficient at frequency $f_{k}$ and $n(t)$ is the total disturbance due to thermal noise and interference. In particular, $n(t)$ is the sum of two terms, say $z_{n}(t)$ and $z_{I}(t)$, where $z_{n}(t)$ is complex additive white Gaussian noise (AWGN) with spectral density $N_{0}$ for each component, and $z_{I}(t)$ is a stationary interference term with power spectral density $S_{\mathrm{I}}(f)$ for each component. Thus, the power spectral density of each component of $n(t)$ is expressed as $S_{\mathrm{N}}(f)=$ $N_{0}+S_{\mathrm{I}}(f)$. The interference is modeled as a zero-mean complex Gaussian process. Considering a cognitive radio framework, it is assumed that $S_{\mathrm{I}}(f)$ is known at the receiver [1], [2], [3].

${ }^{1} \mathrm{~A}$ guard interval between symbols is assumed to avoid inter-symbol interference at the receiver.

${ }^{2}$ The corresponding RF signal power is $E_{\mathrm{p}} /\left(2 T_{\mathrm{s}}\right)$. 


\section{CRB FOR TOA ESTIMATION IN THE PRESENCE OF INTERFERENCE}

In this section, we consider the best achievable accuracy in estimating parameter $\tau$ from the observation of $r(t)$ over an interval $\mathcal{T}_{\text {obs }}$ that comprises the waveform in (2). The length of $\mathcal{T}_{\text {obs }}$ is assumed sufficiently longer than $T_{s}$ to account for the uncertainty on the actual value of $\tau$ and consider the noise outside the interval uncorrelated with the noise inside. We also assume that information on the interference spectral density $S_{I}(f)$ is available from the spectrum awareness engine of a CR [4], [5].

Let $S_{\mathrm{r}}(f, \boldsymbol{\theta})$ represent the Fourier transform of $s_{\mathrm{r}}(t-\tau)$,

$$
S_{\mathrm{r}}(f, \boldsymbol{\theta})=\sum_{k=1}^{K} \alpha_{k} \sqrt{w_{k}} P\left(f-f_{k}\right) e^{-\jmath 2 \pi f \tau},
$$

where $P(f)$ is the Fourier transform of $p(t)$, and $\boldsymbol{\theta} \triangleq$ $\left[\tau a_{1} \cdots a_{K} \phi_{1} \cdots \phi_{K}\right]$ is a vector collecting all the unknown parameters. In computing the CRB for the estimation of $\tau$, two different approaches can be followed. In one case, called joint bounding, the estimation process concerns all the components of $\boldsymbol{\theta}$ and a bound is derived for each of them. In the other case, the interest is focused on $\tau$ while the other components of $\boldsymbol{\theta}$ are viewed as known parameters. This is referred to as conditional bounding [12].

\section{A. Joint Bounding}

As the disturbance $n(t)$ is colored, without loss of generality we can assume that the received signal is first passed through a whitening filter whose frequency response $H(f)$ is such that [13]

$$
|H(f)|^{2}=\frac{1}{S_{\mathrm{N}}(f)} .
$$

Accordingly, the log-likelihood function can be written as

$$
\ln \Lambda(\boldsymbol{\theta})=\Re\left\{\int_{-\infty}^{\infty} x(t) u^{*}(t, \boldsymbol{\theta}) d t\right\}-\frac{1}{2} \int_{-\infty}^{\infty}|u(t, \boldsymbol{\theta})|^{2} d t
$$

where $x(t)=r(t) \otimes h(t)$ is the convolution of $r(t)$ with the impulse response of the whitening filter $h(t)$, and $u(t, \boldsymbol{\theta})=$ $s_{\mathrm{r}}(t-\tau) \otimes h(t)$.

The CRB for TOA estimation is computed as

$$
\operatorname{Var}(\hat{\tau}) \geq\left[\mathbf{J}^{-1}\right]_{1,1}=\mathrm{CRB}
$$

where $\mathbf{J}$ is the Fisher information matrix (FIM) with elements ${ }^{3}$ [14]

$$
[\mathbf{J}]_{m, n}=\Re\left\{\int_{-\infty}^{\infty} \frac{\partial S_{\mathrm{r}}{ }^{*}(f, \boldsymbol{\theta})}{\partial \theta_{m}} S_{\mathrm{N}}^{-1}(f) \frac{\partial S_{\mathrm{r}}(f, \boldsymbol{\theta})}{\partial \theta_{n}} d f\right\} .
$$

From (4) and (8), it is found after some manipulations ${ }^{4}$

$$
\mathbf{J}=\left[\begin{array}{ccc}
\mathrm{J}_{\tau \tau} & \mathbf{J}_{\tau a} & \mathbf{J}_{\tau \phi} \\
\mathbf{J}_{\tau a}^{T} & \mathbf{J}_{a a} & \mathbf{J}_{a \phi} \\
\mathbf{J}_{\tau \phi}^{T} & \mathbf{J}_{a \phi}^{T} & \mathbf{J}_{\phi \phi}
\end{array}\right]
$$

$3 \Re\{x\}$ and $\Im\{x\}$ denote the real and the imaginary parts of $x$, respectively ${ }^{4} \mathbf{A}^{T}$ represents the transpose of $\mathbf{A}$. where

$$
\begin{gathered}
\mathrm{J}_{\tau \tau}=4 \pi^{2} \Re\left\{\sum_{k=1}^{K} \sum_{l=1}^{K} \alpha_{k}^{*} \alpha_{l} \sqrt{w_{k} w_{l}} y_{k, l}(2)\right\} \\
{\left[\mathbf{J}_{\tau a}\right]_{m}=-2 \pi \sqrt{w_{m}} \Im\left\{e^{j \phi_{m}} \sum_{k=1}^{K} \alpha_{k}^{*} \sqrt{w_{k}} y_{k, m}(1)\right\}} \\
{\left[\mathbf{J}_{\tau \phi}\right]_{m}=-2 \pi \sqrt{w_{m}} \Re\left\{\alpha_{m} \sum_{k=1}^{K} \alpha_{k}^{*} \sqrt{w_{k}} y_{k, m}(1)\right\}} \\
{\left[\mathbf{J}_{a a}\right]_{m, n}=\sqrt{w_{m} w_{n}} \Re\left\{e^{j\left(\phi_{n}-\phi_{m}\right)} y_{m, n}(0)\right\}} \\
{\left[\mathbf{J}_{a \phi}\right]_{m, n}=-\sqrt{w_{m} w_{n}} \Im\left\{e^{-j \phi_{m}} \alpha_{n} y_{m, n}(0)\right\}} \\
{\left[\mathbf{J}_{\phi \phi}\right]_{m, n}=\sqrt{w_{m} w_{n}} \Re\left\{\alpha_{m}^{*} \alpha_{n} y_{m, n}(0)\right\}}
\end{gathered}
$$

with

$$
y_{m, n}(i) \triangleq \int_{-\infty}^{\infty} f^{i} S_{\mathrm{N}}^{-1}(f) P^{*}\left(f-f_{m}\right) P\left(f-f_{n}\right) d f,
$$

for $i=0,1,2$ and $m, n=1,2, \ldots, K$.

Inspection of (9) indicates that the FIM can be put in the form of

$$
\mathbf{J}=\left[\begin{array}{ll}
\mathrm{J}_{\tau \tau} & \mathbf{B} \\
\mathbf{B}^{T} & \mathbf{C}
\end{array}\right]
$$

with

$$
\mathbf{B} \triangleq\left[\mathbf{J}_{\tau a} \mathbf{J}_{\tau \phi}\right]
$$

and

$$
\mathbf{C} \triangleq\left[\begin{array}{ll}
\mathbf{J}_{a a} & \mathbf{J}_{a \phi} \\
\mathbf{J}_{a \phi}^{T} & \mathbf{J}_{\phi \phi}
\end{array}\right]
$$

Thus, substituting (17) into (7) yields

$$
\mathrm{CRB}=\left(\mathrm{J}_{\tau \tau}-\mathbf{B C}^{-1} \mathbf{B}^{T}\right)^{-1} .
$$

Equation (20) takes simpler forms under the following special conditions.

1) Disjoint Spectra: Assume $|P(f)|$ is approximately zero outside $-\Delta / 2 \leq f \leq \Delta / 2$. From (16) we have $y_{m, n}(i)=0$ for $m \neq n$, and (10)-(15) become

$$
\begin{aligned}
& \mathrm{J}_{\tau \tau}=4 \pi^{2} \sum_{k=1}^{K}\left|\alpha_{k}\right|^{2} w_{k} \eta_{k}(2), \\
& \mathbf{J}_{\tau a}=\mathbf{0}, \\
& {\left[\mathbf{J}_{\tau \phi}\right]_{m}=-2 \pi w_{m}\left|\alpha_{m}\right|^{2} \eta_{m}(1),} \\
& \mathbf{J}_{a a}=\operatorname{diag}\left\{w_{1} \eta_{1}(0), w_{2} \eta_{2}(0), \ldots, w_{K} \eta_{K}(0)\right\}, \\
& \mathbf{J}_{a \phi}=\mathbf{0}, \\
& \mathbf{J}_{\phi \phi}=\operatorname{diag}\left\{w_{1}\left|\alpha_{1}\right|^{2} \eta_{1}(0), \ldots, w_{K}\left|\alpha_{K}\right|^{2} \eta_{K}(0)\right\},
\end{aligned}
$$

with

$$
\eta_{k}(i) \triangleq \int_{-\infty}^{\infty} f^{i} S_{\mathrm{N}}^{-1}(f)\left|P\left(f-f_{k}\right)\right|^{2} d f, \quad i=0,1,2 .
$$


Thus, substituting (21)-(26) into (20) yields

$$
\mathrm{CRB}=\left(\mathrm{J}_{\tau \tau}-\mathbf{J}_{\tau \phi} \mathbf{J}_{\phi \phi}^{-1} \mathbf{J}_{\tau \phi}^{T}\right)^{-1}=\left(\sum_{k=1}^{K} w_{k} \lambda_{k}\right)^{-1}
$$

with

$$
\lambda_{k}=4 \pi^{2}\left|\alpha_{k}\right|^{2}\left(\eta_{k}(2)-\frac{\eta_{k}^{2}(1)}{\eta_{k}(0)}\right) .
$$

We see that the contribution of each subcarrier to the CRB is determined by the corresponding weight $w_{k}$, the squared channel gain $\left|\alpha_{k}\right|^{2}$, the spectrum of pulse $p(t)$, and the power spectral density $S_{I}(f)$ of the interference around $f_{k}$.

2) Slowly-varying $S_{N}(f)$ : The coefficient $\lambda_{k}$ in (29) can be further simplified assuming $S_{\mathrm{N}}(f) \cong S_{\mathrm{N}}\left(f_{k}\right)=N_{0}+S_{I}\left(f_{k}\right)$ for $\left|f-f_{k}\right| \leq \Delta / 2 \forall k$. In these conditions, (27) becomes

$$
\begin{aligned}
\eta_{k}(i) & \cong \frac{1}{S_{N}\left(f_{k}\right)} \int_{-\infty}^{\infty} f^{i}\left|P\left(f-f_{k}\right)\right|^{2} d f \\
& =\frac{1}{S_{N}\left(f_{k}\right)} \int_{-\infty}^{\infty}\left(f+f_{k}\right)^{i}|P(f)|^{2} d f
\end{aligned}
$$

Then, defining

$$
\beta_{i} \triangleq \frac{1}{E_{\mathrm{p}}} \int_{-\infty}^{\infty} f^{i}|P(f)|^{2} d f \quad i=0,1,2
$$

and bearing in mind that

$$
\int_{-\infty}^{\infty}|P(f)|^{2} d f=E_{\mathrm{p}}
$$

we get

$$
\begin{gathered}
\eta_{k}(2)=\frac{E_{\mathrm{p}}}{S_{N}\left(f_{k}\right)}\left(\beta_{2}+2 f_{k} \beta_{1}+f_{k}^{2}\right) \\
\eta_{k}(1)=\frac{E_{\mathrm{p}}}{S_{N}\left(f_{k}\right)}\left(\beta_{1}+f_{k}\right), \\
\eta_{k}(0)=\frac{E_{\mathrm{p}}}{S_{N}\left(f_{k}\right)} .
\end{gathered}
$$

Finally, substituting (33)-(35) into (29) produces

$$
\lambda_{k}=\frac{4 \pi^{2} E_{\mathrm{p}}\left|\alpha_{k}\right|^{2}\left(\beta_{2}-\beta_{1}^{2}\right)}{N_{0}+S_{\mathrm{I}}\left(f_{k}\right)} .
$$

The physical meanings of $\beta_{2}$ and $\beta_{1}$ are of interest. From (31), we recognize that the former gives the mean square bandwidth of $p(t)$ while the latter represents the skewness of the spectrum $|P(f)|^{2}$. When $|P(f)|$ is an even function, $\beta_{1}$ becomes zero.

Equation (36) indicates that the contribution of the $k$ th subcarrier is proportional to $\left|\alpha_{k}\right|^{2} /\left(N_{0}+S_{\mathrm{I}}\left(f_{k}\right)\right)$. As the channel gain increases and/or the interference spectral density around $f_{k}$ decreases, $\lambda_{k}$ gets larger and the CRB reduces.

\section{B. Conditional Bounding}

Assuming that the components of $\boldsymbol{\theta}$ are all known except for $\tau$, the CRB for TOA estimation can be derived from (7)(8) by considering the estimation of a single parameter. As a result, we get

$$
\mathrm{CRB}=\int_{-\infty}^{\infty}\left|\frac{\partial S_{\mathrm{r}}(f, \boldsymbol{\theta})}{\partial \tau}\right|^{2} S_{\mathrm{N}}^{-1}(f) d f=\left[\mathrm{J}_{\tau \tau}\right]^{-1},
$$

where $J_{\tau \tau}$ is still as in (10). Comparison with (28) reveals that the conditional bound is equal or less than the joint bound. This is intuitively clear because precise information on the nuisance parameters $\left[a_{1} \cdots a_{K} \phi_{1} \cdots \phi_{K}\right]$ is assumed to be available in the former case.

1) Disjoint Spectra and Slowly-varying $S_{N}(f)$ : In this case, $\mathrm{J}_{\tau \tau}$ and $\eta_{2}(2)$ are given by (21) and (33), respectively. Thus, the CRB takes the same form as in the joint bounding case (c.f. (28)):

$$
\mathrm{CRB}=\left(\sum_{k=1}^{K} w_{k} \bar{\lambda}_{k}\right)^{-1}
$$

with

$$
\bar{\lambda}_{k} \triangleq \frac{4 \pi^{2} E_{\mathrm{p}}\left|\alpha_{k}\right|^{2}\left(\beta_{2}+2 f_{k} \beta_{1}+f_{k}^{2}\right)}{N_{0}+S_{\mathrm{I}}\left(f_{k}\right)} .
$$

Note that the difference

$$
\bar{\lambda}_{k}-\lambda_{k}=\frac{4 \pi^{2} E_{\mathrm{p}}\left|\alpha_{k}\right|^{2}\left(\beta_{1}+f_{k}\right)^{2}}{N_{0}+S_{\mathrm{I}}\left(f_{k}\right)}
$$

is positive so that $\bar{\lambda}_{k}>\lambda_{k}$. This agrees with our expectation that conditional bounding gives a lower CRB than joint bounding.

\section{Optimal Weights}

We now concentrate on the optimal assignment of weights that minimizes the CRB. The optimal weights must satisfy constraints on the emitted signal spectrum imposed by regulatory masks (for example, the FCC mask for UWB signals [16]). Let $B(f)$ denote the equivalent baseband version of the power spectral density mask to be met. Then, defining $\boldsymbol{w} \triangleq\left(w_{1}, w_{2}, \ldots, w_{K}\right)^{T}$ and $\boldsymbol{\lambda} \triangleq\left(\lambda_{1}, \lambda_{2}, \ldots \lambda_{K}\right)^{T}$ (c.f. (28) and (38)), the optimal weights are found as the solution of the following optimization problem:

$$
\underset{\boldsymbol{w}}{\operatorname{maximize}} \lambda^{T} \boldsymbol{w}
$$

subject to

$$
\begin{gathered}
\mathbf{1}^{T} \boldsymbol{w} \leq 1 \\
\boldsymbol{w} \succeq \mathbf{0} \\
\boldsymbol{w} \preceq \mathbf{b}
\end{gathered}
$$

where $\mathbf{x} \preceq \mathbf{y}$ means the $i$ th element of $\mathbf{x}$ is smaller than or equal to the $i$ th element of $\mathbf{y} \forall i, \mathbf{1}$ is the vector of all ones, $\mathbf{b} \triangleq\left(b_{1}, b_{2}, \ldots, b_{K}\right)^{T}$, and $b_{k}=B\left(f_{k}\right) \Delta / P_{\mathrm{t}}$ is the normalized emission power constraint on the $k$ th subcarrier.

This is a classical linear programming problem that has the following closed-form solution. Without loss of generality assume that the $\lambda_{k}$ are in decreasing order ${ }^{5}$, i.e., $\lambda_{1}>\lambda_{2}>$

\footnotetext{
${ }^{5}$ The solution is easily extended to the case in which two or more $\lambda_{k}$ are equal.
} 


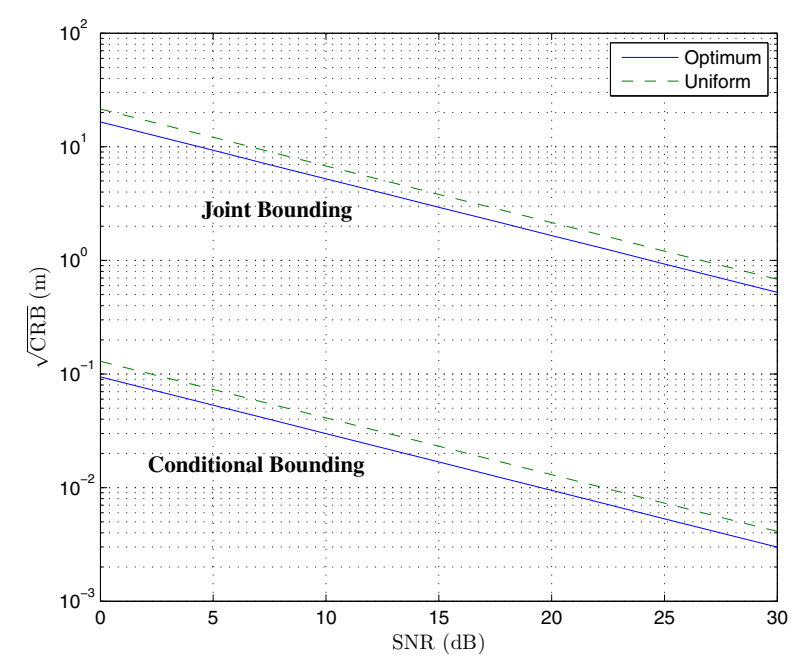

Fig. 1. $\sqrt{\mathrm{CRB}}$ versus SNR for optimal and conventional algorithm in the absence of interference.

$\ldots>\lambda_{K}$. Then, the optimal weights are recursively obtained as

$$
w_{i}^{(\mathrm{opt})}=\min \left\{b_{i}, 1-\sum_{j=1}^{i-1} w_{j}^{(\mathrm{opt})}\right\},
$$

with $w_{1}^{(\mathrm{opt})}=\min \left\{1, b_{1}\right\}$ and $i=2,3, \ldots, K$.

\section{NumericAl RESUlts}

In this section, we provide numerical results that illustrate the impact of the optimal weight selection on the TOA estimation in the presence of interference. We consider a scenario with a subcarrier spacing $\Delta=10 \mathrm{MHz}$ and $K=$ 128 subcarriers. The channel coefficients $\alpha_{k}$ are modeled as independent complex-valued Gaussian random variables with unit average power, and the results are obtained by averaging over 500 independent channel realizations. Pulse $p(t)$ in $(1)$ is modeled as a Gaussian doublet, expressed by

$$
p(t)=A\left(1-\frac{4 \pi t^{2}}{\zeta^{2}}\right) e^{-\frac{2 \pi t^{2}}{\zeta^{2}}},
$$

with $A=\sqrt{\frac{8 E_{\mathrm{p}}}{3 \zeta}}$, where $E_{\mathrm{p}}$ is the pulse energy and parameter $\zeta$ serves to adjust the pulse width. In our experiments, we choose $\zeta=0.4 \mu \mathrm{s}$, which corresponds to a pulse width of about $1 \mu \mathrm{s}$. Parameters $\beta_{1}$ and $\beta_{2}$ in (31) are $\beta_{1}=0$ and $\beta_{2}=\frac{5}{2 \pi \zeta^{2}}$, respectively. The following results are expressed in terms of the square-root of the CRB for the ranging error, which is computed as the product of the square-root of the CRB for TOA, multiplied by the speed of light.

Figure 1 illustrates $\sqrt{\mathrm{CRB}}$ (in meters) versus the signalto-noise ratio (SNR) in the absence of interference for the optimal algorithm (with the weights computed from (45)) and for a conventional algorithm that assigns equal weights to the subcarriers (uniform) in the cases of joint and conditional bounding described in Section III-A and Section III$\mathrm{B}$, respectively. The $\mathrm{SNR}$ is defined as $\mathrm{SNR}=E_{\mathrm{p}} / N_{0}$. It
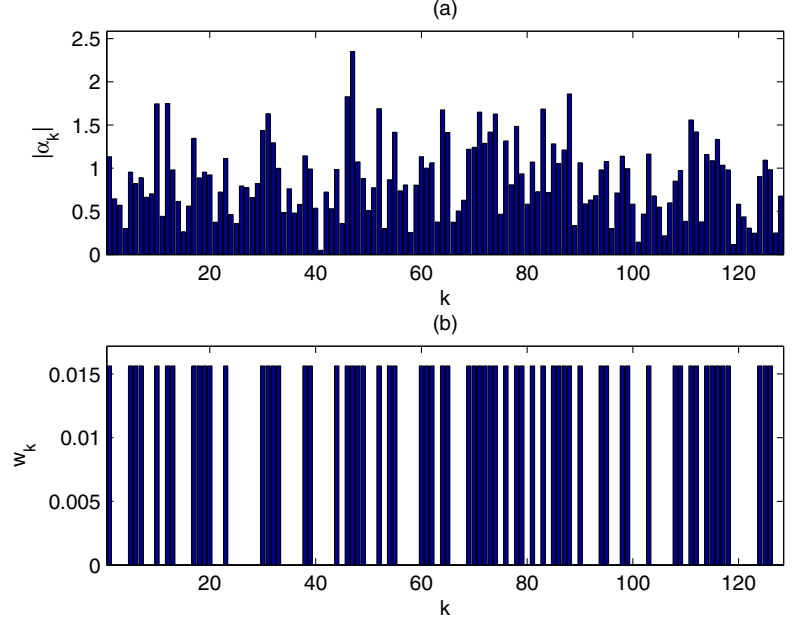

Fig. 2. (a) Channel amplitudes versus subcarrier index. (b) Optimal weights versus subcarrier index.

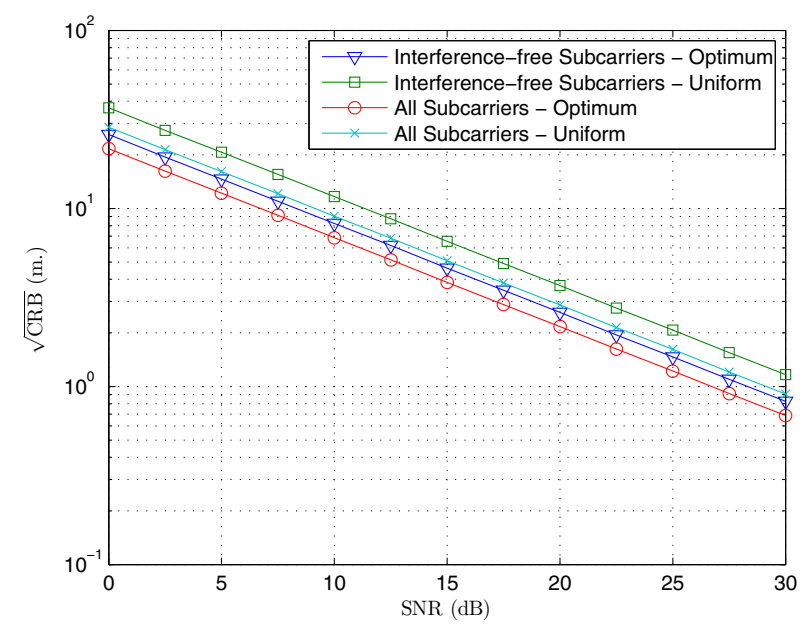

Fig. 3. $\sqrt{\mathrm{CRB}}$ versus SNR for the optimal and conventional (uniform) algorithm in the presence of interference with a flat spectral density in the interval $23 \leq k \leq 106$

is assumed that $w_{k}$ cannot exceed $2 / K$, i.e., $b_{k}=2 / K$ for $k=1, \ldots, K$. It is seen that a gain of about $3 \mathrm{~dB}$ in terms of SNR is obtained with the optimal weights in both cases. In addition, the bounds obtained from conditional bounding are observed to be very low (optimistic), since that technique assumes knowledge of the channel coefficients. Therefore, the following results consider only the joint bounding.

Figure 2 shows a realization of the channel coefficients and the corresponding optimal weights. As expected, the subcarriers with larger channel amplitudes are favored.

Next, we consider the effects of interference. All the system parameters are as before, but $S_{I}(f)$ now takes a constant value $2 N_{0}$ for subcarrier indexes $k$ between 23 and 106 and is zero elsewhere. In Figure 3, the square-root of the CRB is plotted against SNR for two different scenarios. In the first one, an interference avoidance strategy is adopted where the transmitted signal has no power at the subcarriers with 


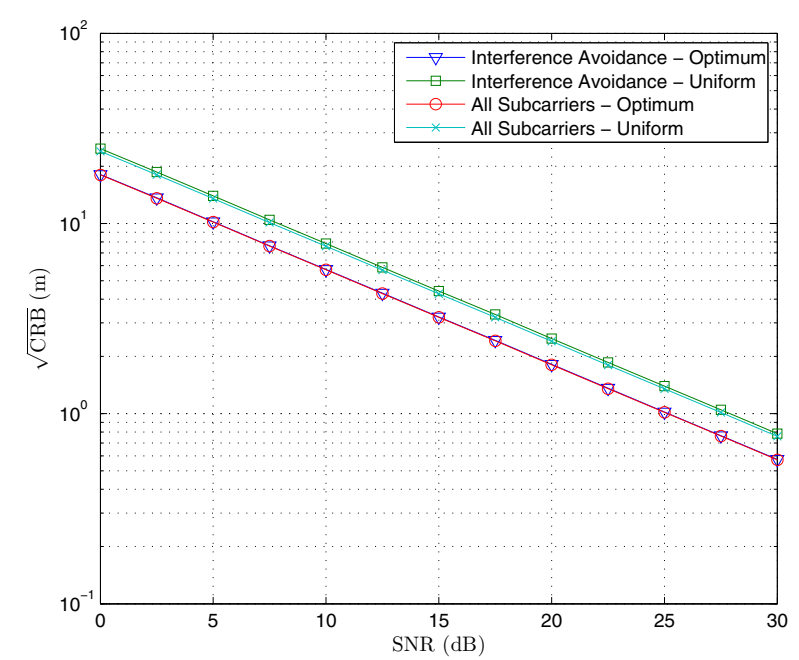

Fig. 4. $\sqrt{\mathrm{CRB}}$ versus SNR for the optimal and conventional (uniform) algorithm in the presence of interference with a flat spectral density in the interval $49 \leq k \leq 80$.

interference, i.e., $w_{k}=0$ for $23 \leq k \leq 106$, while in the second, all the subcarriers can potentially be employed. In both cases, the conventional (uniform) and the optimal algorithm are examined. It can be seen that using all the subcarriers reduces the $\mathrm{CRB}$ with respect to the interference avoidance strategy. However, the improvement becomes insignificant as the number of subcarriers affected by the interference gets small and/or the interference power increases. This is seen in Fig. 4, which shows the square-root of the CRB when the interference spectrum extends from subcarrier 49 to subcarrier 80 with a spectral density of $4 N_{0}$.

Figure 5 illustrates the subcarrier coefficients $\lambda_{k}$ in (36) and the corresponding optimal weights distribution in two scenarios: One uses only the interference-free subcarriers (interference avoidance), whereas the other employs all the subcarriers. As noted from (28) and (45), the subcarriers with large $\lambda_{k}$ values are favored in the optimal spectrum.

\section{CONCLUSIONS}

The cognitive radio paradigm has been applied to TOA measurements. In particular, the CRB for TOA estimation has been computed in the presence of interference. The result has been used to derive the optimal signal power allocation given the measured interference spectrum and the regulatory emission mask. The results imply that the intuitive interference avoidance strategy, which allocates the signal power only on the interference-free subcarriers, is not optimal.

\section{REFERENCES}

[1] J. Mitola and G. Q. Maguire, "Cognitive radio: Making software radios more personal," IEEE Personal Commun. Mag., vol. 6, pp. 13-18, Aug. 1999.

[2] S. Haykin, "Cognitive radio: Brain-empowered wireless communications," IEEE J. Sel. Areas Commun., vol. 23, no. 2, pp. 201-220, Feb. 2005.

[3] Q. Zhi, C. Shuguang, H. V. Poor, and A. Sayed, "Collaborative wideband sensing for cognitive radios," IEEE Signal Processing Magazine, vol. 25, issue 6, pp. 60-73, Nov. 2008.
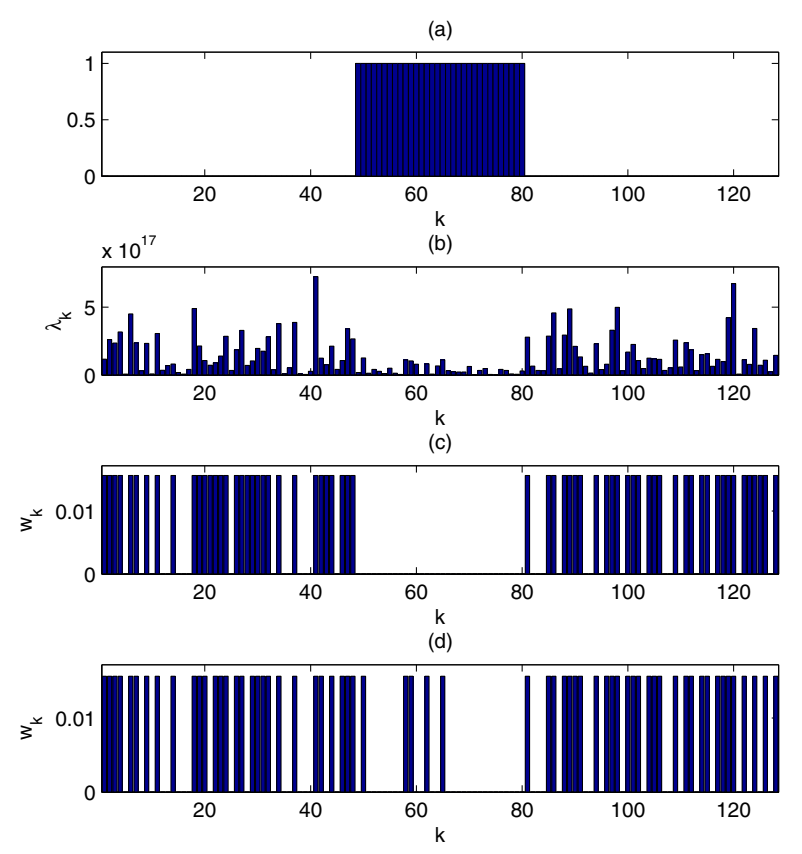

Fig. 5. (a) Spectrum of the interference. (b) Subcarrier coefficient $\lambda_{k}$ versus subcarrier index $k$. (c) Subcarrier weights versus subcarrier index for the optimal algorithm that uses only the interference-free subcarriers (interference avoidance). (d) Subcarrier weights versus subcarrier index for the optimal algorithm that uses all the subcarriers.

[4] H. Celebi and H. Arslan, "Enabling location and environment awareness in cognitive radios," Elsevier Computer Communications (Special Issue on Advanced Location-Based Services), vol. 31, no. 6, pp. 11141125, April 2008.

[5] H. Celebi and H. Arslan, "Cognitive positioning systems," IEEE Trans. Wireless Commun., vol. 6, no. 12, pp. 4475-4483, Dec. 2007.

[6] M. Z. Win and R. A. Scholtz, "Characterization of ultra-wide bandwidth wireless indoor communications channel: A communication theoretic view," IEEE J. Sel. Areas Commun., vol. 20, no. 9, pp. 16131627, Dec. 2002

[7] Z. Sahinoglu, S. Gezici and I. Guvenc, Ultra-Wideband Positioning Systems, New York: Cambridge University Press, 2008.

[8] S. Gezici, Z. Tian, G. B. Giannakis, H. Kobayashi, A. F. Molisch, H. V. Poor, and Z. Sahinoglu, "Localization via ultra-wideband radios: a look at positioning aspects for future sensor networks," IEEE Signal Process. Mag., vol. 22, pp. 70-84, Jul. 2005.

[9] D. Dardari, A. Conti, U. Ferner, A. Giorgetti, and M. Z. Win, "Ranging with ultrawide bandwidth signals in multipath environments," Proc. of IEEE, Special Issue on UWB Technology \& Emerging Applications, vol.97, no. 2, Feb. 2009.

[10] A. Giorgetti, M. Chiani, D. Dardari, R. Piesiewicz, and G. Bruk, "The cognitive radio paradigm for ultra-wideband systems: the European Project EUWB," in Proc. of IEEE Int. Conf. on Ultra-Wideband (ICUWB), Leibniz Universitt Hannover, Germany, Sep. 2008.

[11] T. A. Weiss and F. K. Jondral, "Spectrum pooling: An innovative strategy for the enhancement of spectrum efficiency," IEEE Commun. Mag., vol. 42, no. 3, pp. 814, March 2004.

[12] B. Z. Bobrovsky, E. Mayer-Wolf, and M. Zakai, "Some classes of global Cramér-Rao bounds," The Annals of Statistics, vol. 15, no. 4, pp. 1421-1438, 1987.

[13] H. L. Van Trees, Detection, Estimation, and Modulation Theory, 1st ed. New York, NY 10158-0012: John Wiley \& Sons, Inc., 1968.

[14] S. K. Kay, Fundamentals of Statistical Signal processing: Estimation Theory, Upper Saddle River, New Jersey, USA: Prentice-Hall, 1993.

[15] J. Zhang, R. A. Kennedy, and T. D. Abhayapala, "Cramer-Rao lower bounds for the synchronization of UWB signals," in EURASIP Journal on Wireless Communications and Networking, vol. 3, 2005.

[16] Federal Communications Commission, First Report and Order 02-48, Feb. 2002. 